\title{
An Approach for Detection and Classification of Fruit Disease: A Survey
}

\author{
Zalak R. Barot ${ }^{1}$, Narendrasinh Limbad ${ }^{2}$ \\ ${ }^{1}$ Department of Information Technology, L.J Institute of Engineering \& Technology Ahmedabad 382210, Gujarat, India \\ ${ }^{2}$ Department of Computer Engineering / IT, L.J Institute of Engineering \& Technology, Ahmedabad 382210, Gujarat, India
}

\begin{abstract}
Agriculture is the mother of all cultures. It has played an important role in the development of human civilization. Using image Processing can detect degradation in plant/fruit. Plant fruit disease and leaf disease are crucial causes that which reduce quantity and can degrade the quality of the agricultural products. Currently chemicals are applied to the plants periodically without knowing the requirement of plants. There are some common symptoms of plant like color distortion, abnormal leaf growth, shriveled and damaged pods, stunted growth. Although diseases and insect pests can cause considerable yield losses or bring death to plants and it's also directly affect to human health. These require careful diagnosis and timely handling to protect the crops from heavy losses. In plant, diseases can be found in various parts such as fruit, stem and leaves. This paper represents the survey of various approaches for segmentation method along with feature extraction and classifiers for detection of diseases in fruit and leaf.
\end{abstract}

Keywords: Image Processing, Pre-Processing, Segmentation, Feature Extraction, Classification

\section{Introduction}

India is an agricultural country; wherein about $70 \%$ of the population depends on agriculture. Farmers have wide range of diversity to select suitable Fruit and Vegetable crops[1]. The aim of research in agriculture is to increase the productivity and food quality at reduced expenditure and with increased profit because in India most of the population depends on agriculture. Vegetables and fruits are the most important agricultural products. Agricultural production mainly depends on the quality of seed, soil etc. In order to obtain more valuable products, a product quality control is basically mandatory. The quality of agricultural products may be reduced due to plant diseases. Plant diseases interrupt its vital functions such as photosynthesis, transpiration, pollination, fertilization, germination etc. These diseases are caused by pathogens viz., fungi, bacteria and viruses, and due to adverse environmental conditions. Therefore, the early stage diagnosis plays an important role in plant disease detection. In plant, diseases can be found in various parts such as fruit, stem and leaves.

The classical approach for detection and identification of fruit diseases is based on the naked eye observation by the experts. In some developing countries, consulting experts are expensive and time consuming due to the distant locations of their availability. Automatic detection of fruit diseases is essential to automatically detect the symptoms of diseases as early as they appear on the growing fruits [3].

Currently chemicals are applied to the plants periodically without knowing the requirement of plants. Because of that, healthiness of product may decrease and it will directly affect to human health. There are some common symptoms by which one can come to know about degradation of plant like abnormal leaf growth, color distortion, stunted growth, shriveled and damaged pods. So to protect plant from degradation of quality or heavy losses, these require careful diagnosis and timely handling.
The purpose of this paper is to discuss various techniques for segmentation, feature extraction and classification. It is organized into the following sections. Section 1 gives an introductory part and importance of plant disease detection. Section 2 describes various types of fruit diseases. Section 3 presents a detailed description of basic steps to detect and classify disease on fruit or leaf. Section 4 includes a brief review on Literature. Section 5 concludes this paper.

\section{Types of Disease in Fruit}

There are two type of fruit disease: Biotic and Abiotic. Biotic disease caused by infections agents like fungi, bacteria, viruses and nematodes. Whereas Abiotic disease caused by noninfectious, weather stress, nutrient deficiency, chemical injury and soil factors.

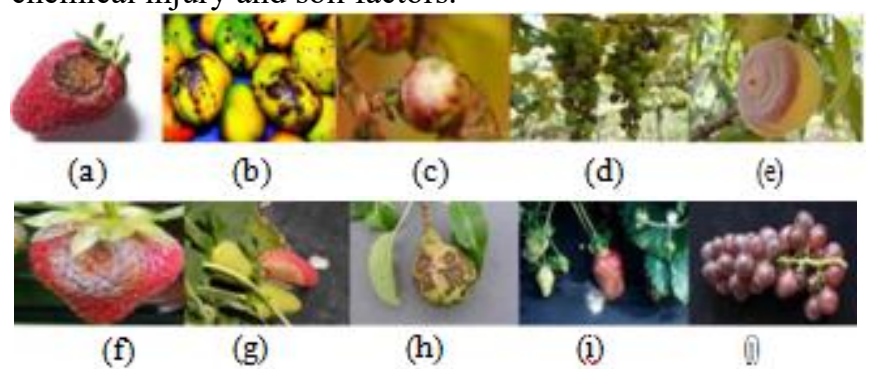

Figure 1: (a) Anthracnose in Strawberry (b) Anthracnose in Mango (c) Powdery Mildew in Strawberry (d) Downey Mildew in Grapes (e) Brown Rot in Pitch (f) Botrytis Rot in Strawberry (g) Gray Mold in Strawberry (h) Scab in pear (i) Sunscald in Strawberry (j) Bitter Rot and Anthracnose in Grapes

\section{Basic Steps for Disease Detection}

In this section, the basic steps for plant disease detection and classification using image processing are shown (Fig. 2). 


\section{International Journal of Science and Research (IJSR) \\ ISSN (Online): 2319-7064}

Index Copernicus Value (2013): 6.14 | Impact Factor (2014): 5.611

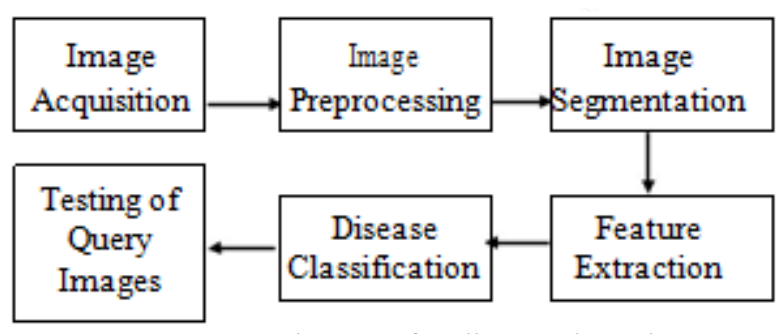

Figure 2: Basic steps for disease detection

\section{a) Image Acquisition}

The images of fruits are captured through the camera. This captured images are in RGB (Red, Green And Blue) form. Color transformation structure for the RGB fruit image is created, after that, for the color transformation structure, a device-independent color space transformation is applied.

\section{b) Image Pre-processing}

To remove noise from image or other object removal, there are various techniques are available. For image clipping, crop the Fruit image to get the interested image region, For smoothing, smoothing filter is used. Image enhancement is done for increasing the contrast, the RGB images into the grey images using colour conversion after that the histogram equalization is applied to distribute the intensities of the images.

\section{c) Image Segmentation}

Segmentation means partitioning of image into various part of same features or having some similarity. The segmentation can be done using various methods like otsu' method, k-means clustering, converting RGB image into HIS model etc.

1] Segmentation using Boundary and spot detection algorithm: For segmentation the RGB image is converted into the HIS model. There are basically two methods to find infected part of fruit, which are Boundary detection and spot detection. For boundary detection the 8 connectivity of pixels are consider and boundary detection algorithm is applied.

2] Threshold Algorithm: Thresholding convert gray-level images into binary images by setting all pixels below some threshold to zero and all pixels above that threshold to one.

The Otsu algorithm is as follows[6]:

i) According to threshold, Separate pixels into two clusters.

ii) Then find the mean of each cluster.

iii) Square the difference between the means.

iv) Multiply the number of pixels in one cluster times the number in the other The infected leaf shows the symptoms of the disease by changing the color of the leaf. Hence the greenness of the leaves can be used for the detection of the infected portion of the leaf. The R, G and B component are extracted from the image. The threshold is calculated using the

Otsu's method. Then the green pixels is masked and removed if the green pixel intensities are less than the computed threshold.
3] K-means clustering: The K-means clustering is used to classify an object based on a set of features into $\mathrm{K}$ number of classes. This classification of object is done by minimizing the sum of the squares of the distance between the object and the corresponding cluster.

The algorithm for $\mathrm{K}$-means Clustering[6]:

i).Pick center of $\mathrm{K}$ cluster, either randomly or based on some heuristic.

ii).Assign each pixel in the image to the cluster that minimizes the distance between the pixel and the cluster center. iii).Again compute the cluster centers by averaging all of the pixels in the cluster. Repeat steps 2 and 3 until convergence is attained.

\section{d) Feature Extraction}

Feature Extraction is used to validate the accuracy and efficiency of image using features like color, texture, shape and etc. There are various techniques to extract feature from image like Global Color Histogram, Color Coherence Vector, Local Binary Pattern and Complete Local Binary Pattern[3].

\section{1) Global Color Histogram (GCH)}

For encoding to the information present in image Global Color Histogram $(\mathrm{GCH})$ is used. It is the simplest approach for that. A GCH is a set of ordered values, for each distinct color, representing the probability of a pixel being of that color. Uniform normalization and quantization are used to avoid scaling bias and to reduce the number of distinct colors.

\section{2) Color Coherence Vector (CCV)}

An approach to compare images based on color coherence vectors are presented in [3]. They define color coherence as the degree to which image pixels of that color are members of a large region with homogeneous color. These regions are referred as coherent regions. Coherent pixels are belongs to some sizable contiguous region, whereas incoherent pixels are not. In order to compute the CCVs, the method blurs and discretizes the image's color-space to eliminate small variations between neighboring pixels. Then, it finds the connected components in the image in order to classify the pixels in a given color bucket is either coherent or incoherent. After classifying the image pixels, CCV computes two color histograms: one for coherent pixels and another for incoherent pixels. The two histograms are stored as a single histogram.

\section{3) Local Binary Pattern (LBP)}

Local Binary Pattern is a simple yet very efficient operator to define local image pattern, and it has reported impressive classification outcomes on representative texture databases Local Binary Pattern has also been adapted by other applications, such as face recognition dynamic texture recognition and shape localization

4) Complete Local Binary Pattern (CLBP)

LBP feature considers only signs of local differences (i.e. difference of each pixel with its neighbors) whereas CLBP feature considers both signs $(\mathrm{S})$ and magnitude $(\mathrm{M})$ of local differences as well as original center gray level (C) value. CLBP feature is the combination of three features, namely

\section{Volume 4 Issue 12, December 2015}




\section{International Journal of Science and Research (IJSR) \\ ISSN (Online): 2319-7064}

Index Copernicus Value (2013): 6.14 | Impact Factor (2014): 5.611

CLBP_S, CLBP_M, and CLBP_C. CLBP_S is the same as the original LBP and used to code the sign information of local differences. CLBP $M$ is used to code the magnitude information of local differences . CLBP_C is used to code the information of original center gray level value.

\section{e) Classification}

There are various technique for classification:

Artificial Neural Network, Backbone Propagation Neural Network, Feed forward Back propagation Neural Network, Probabilistic Neural Network, Support Vector Machine, Multiclass Support Vector Machine and etc.

Some of them are as below:

1) Artificial Neural Network (ANN):

After feature extraction is done, the learning database images are classified by using neural network. These feature vectors are considered as neurons in ANN. The output of the neuron is the function of weighted sum of the inputs. The back propagation algorithm, modified SOM; Multiclass Support vector machines can be used.

2) Backbone Propagation Neural Network (BPNN): BPNN algorithm is used in a recurrent network. Once trained, the neural network weights are fixed and can be used to compute output values for new query images which are not present in the learning database.

3) Support Vector Machine (SVM):

A support vector machine constructs a hyper-plane or set of hyper-planes in a high- or infinite-dimensional space, which can be used for classification, regression, or other tasks.SVM is supervised learning model with associated learning algorithms that analyze data and recognize patterns, used for classification and regression analysis. Given a set of training examples, each marked for belonging to one of two categories, an SVM training algorithm builds a model that assigns new examples into one category or the other, making it a non-probabilistic binary linear classifier.

f) Testing of query images :

After getting the weight of learning database, then testing of query image is done. The fig. 3 shows the flowchart for the testing of query image using the neural network techniques[6].

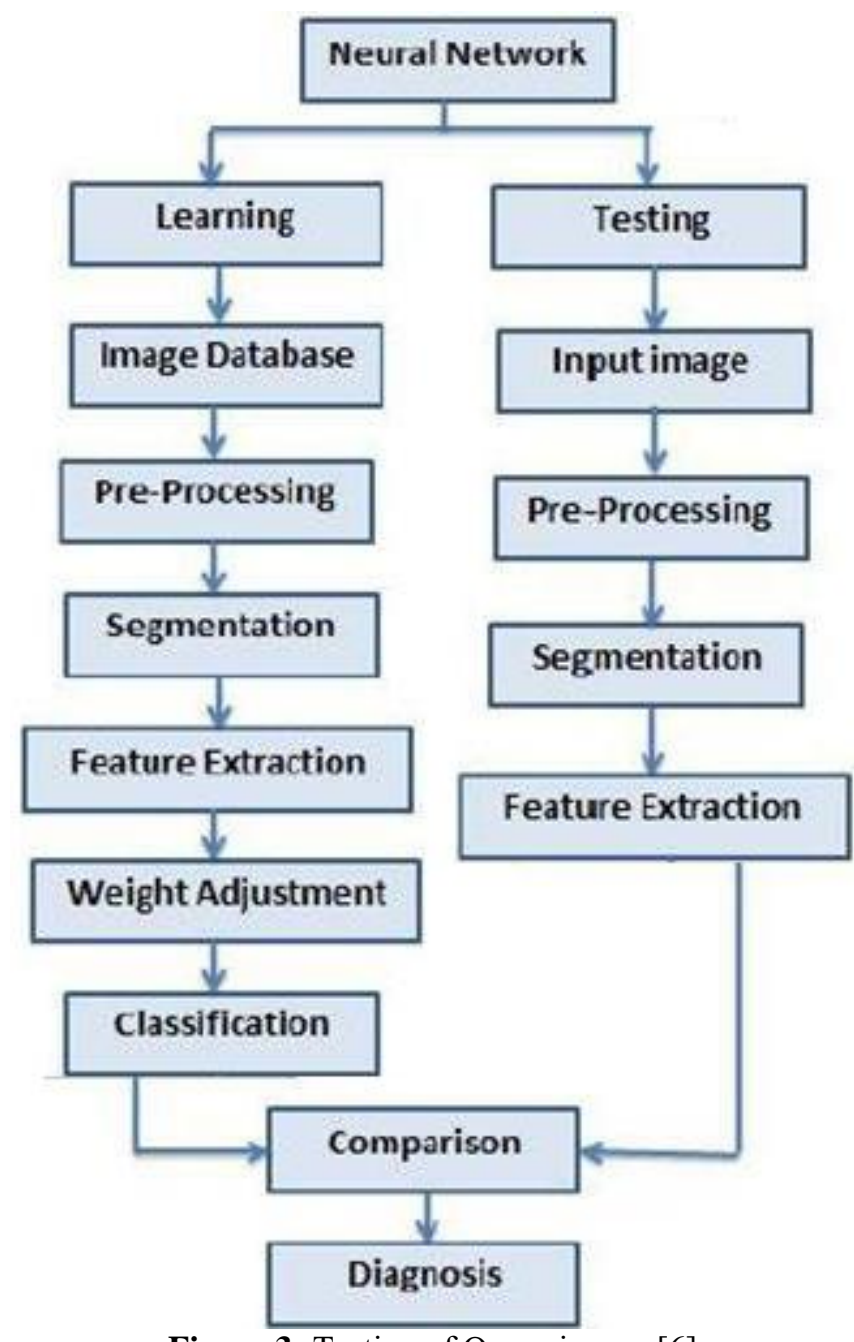

Figure 3: Testing of Query images[6]

\section{Literature Review}

An enough literature is available for plant/fruit disease detection and also classifies using various approaches.

Suhaili Kutty et. al[2] proposed the process to classify Anthracnose and Downey Mildew, watermelon leaf diseases. For this Region of Interest need to be identified from infected leaf sample based on RGB color component. Then to reduce noise and for segmentation Median Filter is used. And for classification, Neural Network Pattern Recognition Toolbox is used. Proposed method achieved $75.9 \%$ of accuracy based on its RGB mean color component.

S. Dubey and R. Jalal [3] explored the concept of detection and classification of apple fruit diseases, namely, scab, apple rot and apple blotch.For that, segmentation is done using $\mathrm{K}$ means clustering technique. Then features are extracted from the segmented image. For classification Multiclass Support Vector Machine (SVM) is used.

The goal of Sanjiv Sannakki et.al[4] is to diagnose the disease using image processing and artificial intelligence techniques on images of grape plant leaf. They classify mainly two diseases, downy mildew and powdery mildew of grape leaf. Masking is used to remove background to improve accuracy. For preserving information of affected portion of leaf, Anisotropic Diffusion is used. Segmentation

\section{Volume 4 Issue 12, December 2015}




\section{International Journal of Science and Research (IJSR) \\ ISSN (Online): 2319-7064 \\ Index Copernicus Value (2013): 6.14 | Impact Factor (2014): 5.611}

is carried out using k-means clustering method. After segmentation, Feature Extraction take place by calculating Gray Level Co-occurrence Matrix. And finally classification is done using Feed Forward Back Propagation Network classifier. They have used only Hue feature which gives more accurate result.

Meunkaewjinda et. al [5] presents automatic plant disease diagnosis using multiple artificial intelligent techniques. They focus on a system having composed of only three main parts which are (1) Grape leaf color extraction,

(2) Grape leaf disease color extraction and (3) Grape leaf disease classification. For Grape leaf color extraction, they enhanced image using anisotropic diffusion. Then $\mathrm{H}$ and $\mathrm{B}$ components are used from HIS and LAB color space respectively. After that Back Propagation neural network is applied to extract grape leaf color from other background color. In Grape leaf disease color extraction, to reduce illumination effects, $\mathrm{A}, \mathrm{U}$, and $\mathrm{Cr}$ components are applied from $\mathrm{LAB}, \mathrm{UVL}$ and $\mathrm{YCbCr}$ color space respectively. Then SVM classification is used for better segmentation compare to neural network. And finally for Grape leaf disease classification process, some irrelevant pixels are eliminated using thresholding, and $\mathrm{H}$ and $\mathrm{Cr}$ components from HIS and YCbCr color space respectively, are applied to extract most important color feature of grape leaf disease. MSVM classification is used for Grape leaf disease classification.

Monika Jhuria et. al[6] investigated an approach of image processing for detection of disease and the fruit grading. They have used artificial neural network for classification of disease. They consider three feature vectors, namely, color, textures and morphology. Among all, morphological feature gives better result. It can detect two disease of grape which are Black Rot and Powdery Mildew and two of apple which are Apple Scab and Rot. Two methods are used for fruit grading which are spread of disease and automated calculation of mango weight.

Sachin Khirade and A. B. Patil[7] discussed about the main steps of image processing to detect disease in plant and classify it. It involves steps like image acquisition, image pre-processing, image segmentation, feature extraction and classification. For segmentation, methods like, otsu's method, converting RGB image into HIS model and kmeans clustering are there. Among all, k-means clustering method gives accurate result. After that, feature extraction is carried out like, color, texture, morphology, edges etc. Among this, morphology feature extraction gives better result. After feature extraction, classification is done using classification methods like Artificial Neural Network and Back Propagation Neural Network.

Ms. Kiran R. Gavhale et al.[8] presented number of image processing techniques to extract diseased part of leaf. For Pre-processing, Image enhancement is done using DCT domain and color space conversion is done. After that segmentation take place using k-means clustering method. Feature extraction is done using GLCM Matrix. For classification of canker and anthracnose disease of citrus leaf, SVM with radial basis kernel and polynomial kernel is used.
Zulkifli Bin Husin et al.[9] explored a system to detect disease on chili leaf. Health status of leaf is going to check. So chemicals only applied if it is affected by any disease, which is inexpensive technique. In this, preprocessing operations are done which are Fourier Filtering, Edge Detection and Morphological Operations. Input image is also enhanced to preserve information of affected pixels. For reducing effect of illumination, they used color space. Using this technique, unnecessary use of harmful chemicals in plant is reduced and ensure a healthier environment and provide high quality of chili.

\section{Conclusion}

This paper conclude different segmentation, feature extraction and classification techniques for plant disease detection using its leaf or fruit. Each and every technique has some merit and demerits. Based on requirement we have to use method for that. Here we had seen different segmentation techniques with its merits and demerits no such segmentation technique applicable in all images so use any of technique which is suitable for our application. Also seen different classification techniques with its merit and demerits. Among different classifier ANN and SVM give better accuracy then other classifier.

\section{References}

[1] Jayamala K. Patil, Raj Kumar, "ADVANCES IN IMAGE PROCESSING FOR DETECTION OF PLANT DISEASES", Journal of Advanced Bioinformatics Applications and Research, pp 135-141, 2011

[2] Suhaili Beeran Kutty, Noor Ezan Abdullah, Dr. Hadzli Hashim, A'zraa Afhzan Ab Rahim, Aida Sulinda Kusim,Tuan Norjihan Tuan Yaakub, Puteri Nor Ashikin Megat Yunus, Mohd Fauzi Abd Rahman, "Classification of Watermelon Leaf Diseases Using Neural Network Analysis,"IEEE, Business Engineering and Industrial Applications Colloquium (BEIAC), Langkawi, pp 459 464, 2013

[3] Shiv Ram Dubey, Anand Singh Jalal," Detection and Classification of Apple Fruit Diseases using Complete Local Binary Patterns," IEEE Computer and Communication Technology (ICCCT), 2012 Third International Conference on Computer and Communication Technology, Allahabad, pp 346-351, 2012

[4] Sanjeev S. Sannaki, Vijay S. Rajpurohit, V B Nargund,PallaviKulkarni, "Diagnosisand Classification of Grape Leaf Diseases using Neural Network," IEEE , Tiruchengode, pp 1-5, 2013

[5] Meunkaewjinda, P.Kumsawat, K. Attakitmongcol, A. Srikaew, "Grape leaf disease detection from color imagery using hybrid intelligent system," IEEE, $5^{\text {th }}$ International Conference on , Krabi , pp 513-516, 2008

[6] Monika Jhuria, Ashwani Kumar, Rushikesh Borse, "Image processing for smart farming: Detection of disease andfruitgrading," IEEE, Second International Conference on Image Processing, Shimla, pp 521 - 526, 2013

[7] Sachin D. Khirade, A. B. Patil, "Plant Disease Detection Using Image Processing," IEEE, International 


\section{International Journal of Science and Research (IJSR) \\ ISSN (Online): 2319-7064}

Index Copernicus Value (2013): 6.14 | Impact Factor (2014): 5.611

Conference on Computing Communication Control and Automation, Pune, pp 768-771, 2015

[8] Ms. Kiran R. Gavhale, Prof. Ujwalla Gawande, Mr. Kamal O. Hajari, "Unhealthy Region of Citrus Leaf Detection Using Image Processing Techniques," IEEE, International Conference for Convergence of Technology, Pune, pp 1-6, 2014

[9] Zulkifli Bin Husin, Abdul Hallis Bin Abdul Aziz, Ali Yeon Bin Md Shakaff, Rohani Binti S Mohamed Farook, "Feasibility Study on Plant Chili Disease Detection Using Image Processing Techniques," IEEE, Third International Conference on Intelligent Systems Modelling and Simulation, Kota Kinabalu, pp 291-296, 2012 